and pressure measurements were obtained before giving the frusemide intraveñously and the changes were followed over a six-hour period in 20 patients. Measurement of dead space-tidal volume ratio and venous admixture was made in 11 patients. Changes in gas exchange were correlated with change in cardiac output.

Frusemide initially produced a large diuresis and a small but significant fall in arterial oxygen tension. This correlated with a fall in cardiac output and was associated with an increase in venous admixture. Four and six hours after frusemide, when the patients had a large negative fluid balance, there was no significant difference from pretreatment values in arterial oxygen tension, carbon dioxide tension, dead space-tidal volume ratio or venous admixture. It is suggested that frusemide does not cause a rapid reduction in the volume of pulmonary oedema, and that the changes observed acutely are not due to change in pulmonary oedema but to alteration in the haemodynamic state.

\section{MEASUREMENT OF MYOCARDIAL CONTRACTILITY}

M. I. M. NOBLE This communication summarizes a half-day meeting held by the Royal Society of Medicine on 28 June 1971. For the purposes of this meeting a change of myocardial contractility was defined as a change in the mechanical performance of the heart which is not due to a change in the length of the muscle fibres (Frank-Starling mechanism) and is not directly due to a change in the load on the muscle.

B. R. Jewell explained why valid measurement of this property of the myocardium has not proved possible in isolated heart muscle, the major problems being (1) the lack of agreement on the correct model to be adopted for heart muscle, and (2) the dependence of contractility on time during the contraction.

K. B. Saunders explained why any attempt to apply concepts derived from isolated heart muscle to the intact heart were bedevilled by the inexactitude of the geometric interrelationship between force and ventricular pressure, velocity of shortening and blood flow, muscle length and ventricular volume. However, if end-diastolic volume and aortic pressure are controlled by right heart bypass and aortic resisters, it was shown by J. H. Mitchell that changes in contractility could be demonstrated by determining the relationship to end-diastolic volume of stroke volume, stroke work or ejection fraction. This solution of the problem appeared attractive to physiologists but not to clinicians who need to assess contractility in the intact animal. It was for this reason that a number of 'indices of contractility' had been advocated. These were reviewed by G. van den Bos, who pointed out that while the maximum rate of rise of left ventricular pressure ( $\mathrm{LV} \mathrm{dp/dt} \mathrm{max)} \mathrm{and} \mathrm{the} \mathrm{maximum} \mathrm{accelera-}$ tion of blood from the left ventricle had proved useful in the hands of some authors, neither these nor any other indices had been shown to be valid in all circumstances. There were a number of contributions describing efforts to assess myocardial contractility in animals and patients, but the general consensus of the meeting was that, at the present time, the problems in the measurement of myocardial contractility are far from being solved.

\section{RADIOGRAPHIC CENTILES OF LUNG AND HEART GROWTH \\ Patterns of Growth}

G. SIMON and J. M. TANNER Standard centiles for lung width, lung length, and heart transverse diameter have been constructed for boys and girls from age 6 to 19 based on serial chest radiographs taken annually on 84 boys and 78 girls. Mean velocity curves for these dimensions are also presented. The age at peak velocity for lung width and heart diameter coincides with age at peak height velocity; peak velocity for lung length occurs some six months later. The lungs are exceptional in that the adolescent growth spurt is of the same magnitude in both sexes and the girls' mean values do not exceed the boys' even in adolescence. At age 6 heart diameter is $80 \%$ of its adult value whereas lung width is $66 \%$ and lung length $63 \%$.

\section{Use of Lung Centile Charts in Asthma}

NICOLA COINNOLLY Changes in measurements in the chest radiographs of a series of 97 children with asthma are shown. The children were divided into three groups on the basis of their chest radiographs: normal $(78 \%)$, simple overinflation with normal vessels $(11 \%)$, and overinflation with large hilar vessels relative to the intrapulmonary vessels $(10 \%)$. In the last two groups the lung length was increased in relation to the centile charts of the normal children. In the third group the ratio of lung length to lung width was greater than 1.0 in $60 \%$, a finding uncommon in normal children.

The relation of respiratory function tests and clinical features to the $x$-ray appearances is shown. Apart from a tendency for the very severe cases to fall into the third radiological group, correlation was poor and some of the worst cases had a normal chest radiograph.

\section{Use of Lung Centile Charts in Cystic Fibrosis}

M. B. MEARNS A series of patients with cystic fibrosis seen at the age of 5 years or more and followed up for a minimum of five years was studied, and measurements were made on the annual radiographs as described above. These measurements were then plotted on the lung centile charts of the normal children to try to detect any abnormalities of lung growth associated with or due to the disease. Patients were grouped on combined clinical and radiological criteria as described by Jackson and Young. The height and lung percentiles were correlated with the clinical course. Changes in serial measurements of ventilatory capacity over a minimum period of five years are also correlated with the lung centile changes, and the pattern of change is discussed. 